\title{
Diffusion Driven Concerted Motion of Surface Atoms: Ge on Ge(001)
}

\author{
H. J. W. Zandvliet, T. M. Galea, * E. Zoethout, and Bene Poelsema \\ Department of Applied Physics and MESA+ Research Institute, University of Twente, P.O. Box 217, \\ 7500 AE Enschede, The Netherlands
}

(Received 20 July 1999)

\begin{abstract}
The diffusion of Ge dimers on the Ge(001) surface has been studied with scanning tunneling microscopy. We have identified three different diffusion pathways for the dimers: diffusion of on-top dimers over the substrate rows, diffusion across the substrate rows, and diffusion of dimers in the trough. We report on a heretofore unknown phenomenon, namely, diffusion driven concerted motion of substrate atoms. This concerted motion is a direct consequence of the rearrangement of substrate atoms in the proximity of the trough dimer adsorption site.
\end{abstract}

PACS numbers: 68.35.Fx, 61.16.Ch, 68.35.Bs

A detailed understanding of the mechanisms and energetics involved when atoms or small clusters, such as dimers, diffuse over surfaces is of great fundamental and technological interest with important implications for the development of microscopic models for epitaxial growth and etching. During crystal growth, for example, deposited atoms diffuse over the surface until they become incorporated in the lattice. During its journey from the initial deposition site to its final bound location the atom visits a number of substrate sites. Thermally activated diffusion from substrate site to substrate site can occur either via a simple hop mechanism or via an exchange mechanism. The latter mechanism was initially proposed by Bassett and Webber [1] and its existence was experimentally confirmed for $\mathrm{Pt} / \mathrm{Pt}(001)$ by Kellogg and Feibelman [2] and for $\operatorname{Ir} / \operatorname{Ir}(001)$ by Chen and Tsong [3]. Here we will concentrate on the diffusion of $\mathrm{Ge}$ on the $\mathrm{Ge}(001)$ surface. When $\mathrm{Si}$ or $\mathrm{Ge}$ atoms are deposited on $\mathrm{Si}(001)$ or $\mathrm{Ge}(001)$ surfaces at room temperature the atoms are so mobile that most of them combine quickly to form dimers. The four plausible adsorption sites for a dimer on the (001) substrate are shown in Fig. 1. Two of these configurations (A and B) consist of isolated dimers directly adsorbed on top of the substrate dimer rows and the other two configurations $(C$ and $\mathrm{D})$ are isolated dimers lying in the trough between the substrate dimer rows. The mobility of these dimers is severely limited at room temperature, especially for the dimers on the $\mathrm{Si}(001)$ substrate [4-11]. The preferential diffusion pathway for $\mathrm{Si}$ dimers on $\mathrm{Si}$ and $\mathrm{Ge}(001)$ surfaces is diffusion of on-top dimers along the substrate dimer row direction $[4,5,7,9]$. However, across dimer row diffusion [6-9] as well as diffusion of trough dimers along the trough direction has been observed for Si dimers on $\mathrm{Si}$ and $\mathrm{Ge}(001)$ surfaces, too. Besides the various diffusion pathways of dimers on the (001) surfaces Zhang et al. [10] have shown that $\mathrm{Si}$ on-top dimers also rotate back and forth between two nearly degenerate states (A and B). In this Letter, we have identified three diffusion pathways for Ge dimers on the $\mathrm{Ge}(001)$ surface: diffusion of on-top dimers over the substrate rows, diffusion across the substrate rows, and diffusion of dimers in the trough. We show that all above mentioned diffusion pathways including a rotational mode are already active at room temperature. However, our most intriguing observation is that during diffusion of a trough dimer along the trough or across the substrate dimer rows also the substrate atoms in the proximity of the diffusing dimer exhibit a heretofore unknown concerted motion. In retrospect, the occurrence of this concerted motion is not surprising at all but is a direct consequence of the rearrangement of substrate atoms in the proximity of an adsorbed trough dimer.

The experiments were performed in an ultrahigh vacuum (UHV) system equipped with a scanning tunneling microscope (STM). The base pressure of the system is $\sim 5 \times 10^{-11}$ mbar. The nearly intrinsic and nominally flat $\mathrm{Ge}(001)$ samples were cleaned by various cycles of $800 \mathrm{eV}$ $\mathrm{Ar}^{+}$sputtering and annealing at $1100 \mathrm{~K}$ [12]. After equilibration to room temperature $\sim 0.5 \%-1 \%$ of a monolayer of Ge was deposited from a thoroughly degassed, heated wafer at $3 \times 10^{-4}$ monolayers per second (ML/s). Subsequently, the samples were transferred to the STM for imaging. The complete procedure of deposition and transferring into STM takes about $10 \mathrm{~min}$.

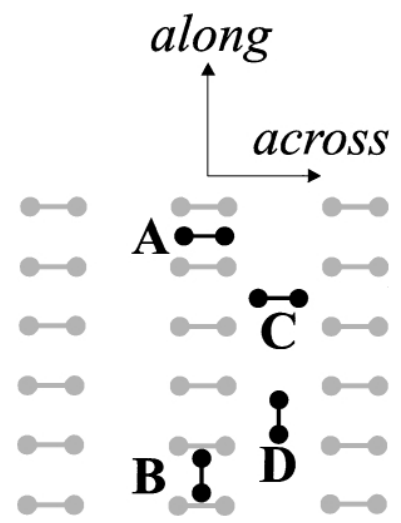

FIG. 1. Schematic diagram of the configurations of isolated adsorbed Ge dimers on Ge(001). Gray dumbbells represent the substrate dimers and black dumbbells represent the adsorbed dimers. 
It is common knowledge that at room temperature the semiconductor group IV (001) surfaces, i.e., Si and $\mathrm{Ge}(001)$, exhibit a $(2 \times 1)$ reconstruction: surface atoms dimerize, eliminating one dangling bond per atom to lower the surface free energy. Two different dimers are observed: symmetric dimers and asymmetric dimers. The symmetric dimers lie in the surface plane, whereas the asymmetric dimers have one atom that buckles out of the surface plane and one atom that buckles inwards. The asymmetric dimer registry shows up in filled state images (sample bias $-1.6 \mathrm{~V}$ ) as a zigzag line running along the substrate rows, because the asymmetric dimers exhibit an antiferromagnetic ordering along the substrate rows. In-phase buckling of the adjacent substrate row results in a $p(2 \times 2)$ reconstruction, whereas out-of-phase buckling gives rise to a $c(4 \times 2)$ reconstruction (see Figs. 2 and $3)$. The nonzigzag substrate dimer rows are comprised of symmetric or at least symmetric appearing dimers (probably the symmetric dimers observed in STM images are just asymmetric dimers that rapidly switch between the two buckled states) [13-15].

When $\sim 0.01 \mathrm{ML}$ of Ge is deposited onto a $\mathrm{Ge}(001)$ surface at room temperature, isolated dimers as well as larger clusters are found. Isolated dimers are found at on-top positions as well as trough positions (Figs. 2 and $3)$. The orientation of the dimer bond of the isolated dimer is either along the substrate dimer bonds ( $\mathrm{A}$ and $\mathrm{C}$ configurations) or perpendicular to the substrate dimer bond (B and D configurations) [16]. Interestingly, Qin and Lagally [17] have suggested recently that the $\mathrm{C}$ Ge dimers on $\mathrm{Si}(001)$ are actually adatom pairs rather than ordinary dimers. They base their conclusion on a detailed analysis of STM images taken at different sample biases. The most remarkable difference with ordinary ad-dimers is that the $\mathrm{C}$ features are quite faint in filled-state images, while ordinary ad-dimers are bright in filled-state images. Although we observe the same contrast difference in our STM observations we will label the $\mathrm{C}$ feature in the trough as a $\mathrm{C}$ dimer, realizing that this $\mathrm{C}$ feature can also be identified as an adatom pair rather than an ordinary dimer. Most of the isolated dimers we have observed in our images are either positioned on-top (configuration B) or in between (configuration C) the substrate rows (see Figs. 2 and 3). The images in Fig. 2 are empty-state images, whereas the images in Fig. 3 are filled-state images. On-top dimers are bright in filled- and empty-state images, whereas the trough dimers appear much brighter in the empty-state images than in the filled-state images. Often the $\mathrm{C}$ dimers cluster into diluted strings running along $\langle 130\rangle$ directions. In contrast to $\mathrm{Si}$ and $\mathrm{Ge}$ on $\mathrm{Si}(001)$ we did not find diluted $\mathrm{CC}$ cluster running perpendicular to the substrate rows, i.e., along $\langle 110\rangle$ directions. To gain detailed insight into the diffusion of Ge dimers on $\mathrm{Ge}(001)$, we have acquired many consecutive STM images of the same area of the surface. As an example a sequence of STM images showing the dynamic behavior of a $\mathrm{Ge}$ dimer on $\mathrm{Ge}(001)$ is pre-
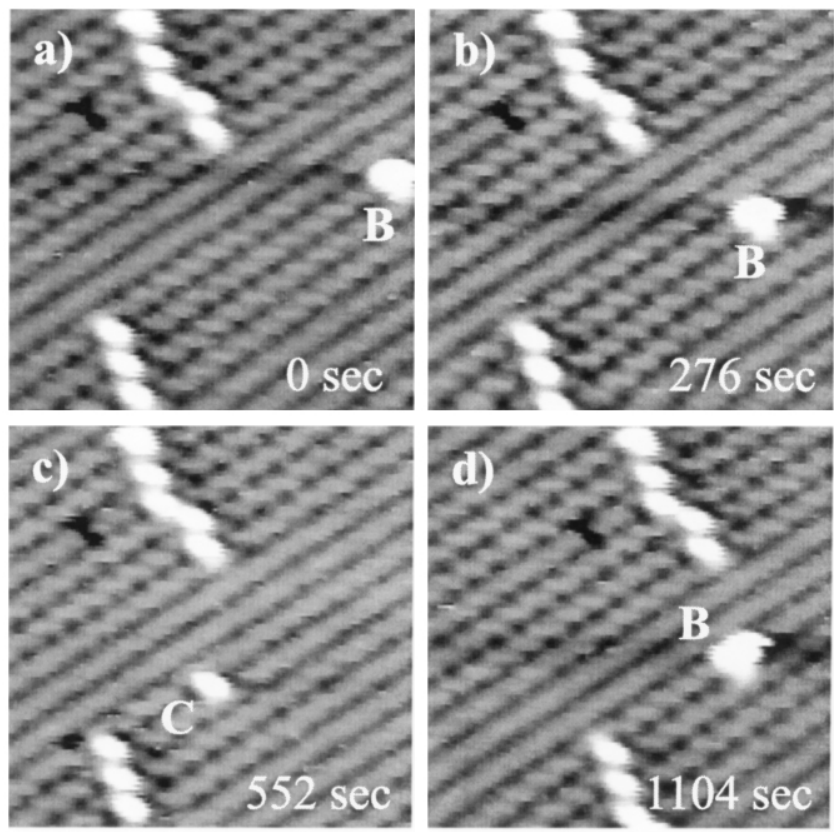

FIG. 2. Successive empty-state scans of the same area $(100 \AA \times 100 \AA)$ of a $\mathrm{Ge}(001)$ surface after the deposition of $\sim 1 \%$ of a monolayer Ge. The time elapsed is depicted in the images. (a),(b) Diffusion of an on-top dimer (B) along a substrate dimer row. (b),(c) The on-top dimer (B) jumps to a trough position (C). (c),(d) The trough dimer (C) jumps to an on-top position of an adjacent substrate row (B) (sample bias $+1.6 \mathrm{~V}$ and tunneling current $0.7 \mathrm{nA}$ )

sented in Figs. 2 and 3. In Fig. 2 a B-type on-top dimer diffuses over the substrate dimer row. In image 2(c) the dimer has hopped to a trough position, while in image 2(d) it hops to an on-top position of the neighboring dimer row. Note that here the transformation from the on-top site to the trough site (and vice versa) goes along with the rotation of the dimer as well. In images 3(a) and 3(b) the same B on-top dimer jumps to a trough position, where it starts to diffuse along the trough. Interestingly, after the hop from the on-top position [image 3(a)] to the trough position [image 3(b)] the complete dimer row where the on-top dimer was positioned becomes buckled. In the next images the trough dimer first migrates two atomic positions towards the CC cluster in the lower left [image 3(d)] and subsequently it migrates one position towards the CC cluster [image 3(e)]. In the final image [3(f)] it moves one position away from the CC cluster. The most interesting observation is the concerted motion of substrate atoms during diffusion [images 3(a) and 3(b) and 3(e) and 3(f)]. As has been discussed by Wulfhekel et al. [7] and Qin and Lagally [17] the CC clusters and the isolated C dimers (or adatom pairs) locally pin a $c(4 \times 2)$ reconstruction. The two substrate dimer rows adjacent to the trough dimer are buckled in this $c(4 \times 2)$ registry. From image 3(d) to image 3(e) the trough dimer has moved one position towards the CC cluster in the lower left. The number of dimers on the two substrate dimer rows trapped between the $\mathrm{C}$ dimer 


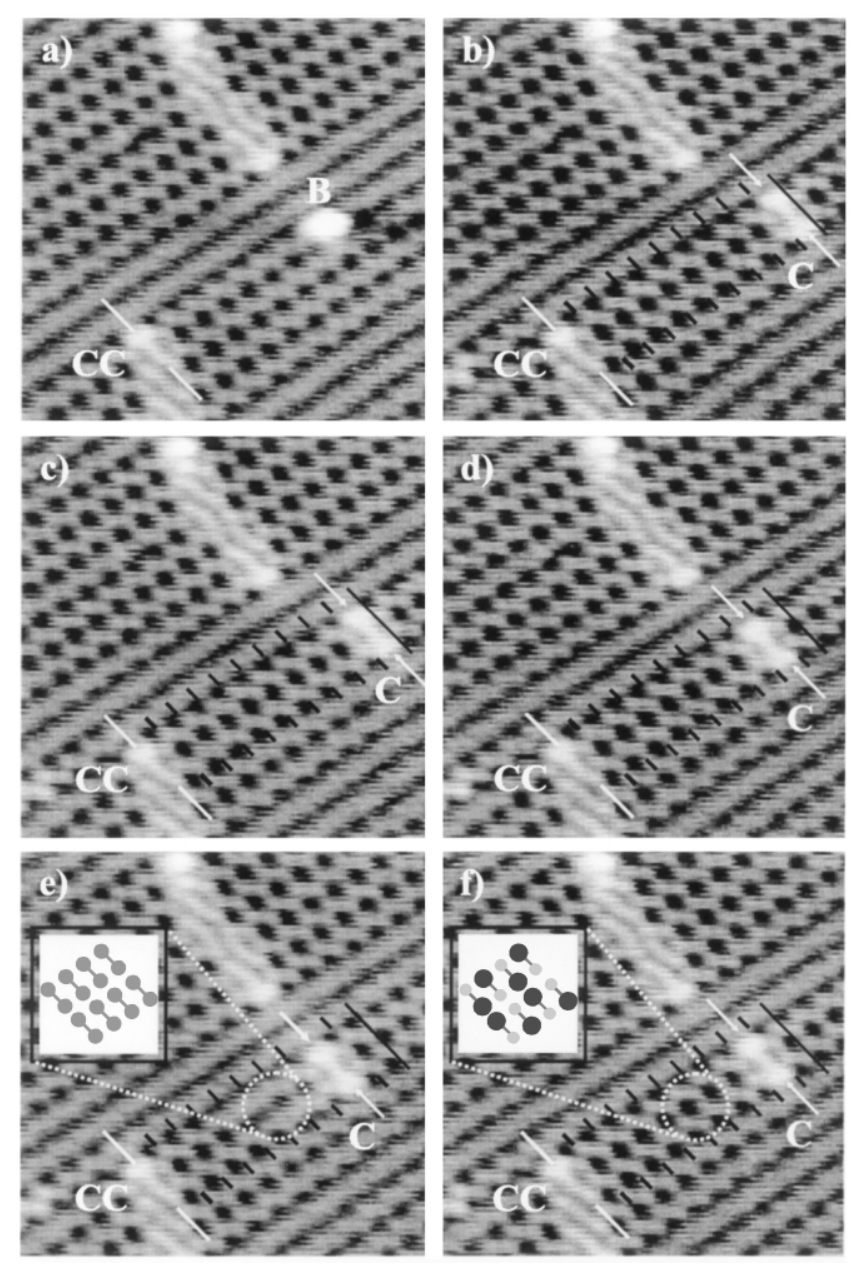

FIG. 3. Successive filled-state scans of the same area $(100 \AA \times 100 \AA)$ of a $\mathrm{Ge}(001)$ surface after the deposition of $\sim 1 \%$ of a monolayer Ge. The time lapse between successive images is $276 \mathrm{~s}$. The spacing between adjacent black markers is two dimer positions, i.e., $8 \AA$. The white markers and the white arrows indicate the positions of the $\mathrm{CC}$ cluster and the $\mathrm{C}$ dimer, respectively. (a),(b) An on-top dimer (B) jumps into the trough (C). (c),(d) The trough dimer jumps two positions towards the cluster. (d),(e) The trough dimer hops one position towards the $\mathrm{CC}$ cluster. (e),(f) The trough dimer jumps one position away from the CC cluster. The insets in images (e) and (f) show the $(2 \times 1)$ and $c(4 \times 2)$ buckling registry, respectively (sample bias $-1.6 \mathrm{~V}$ and tunneling current $0.7 \mathrm{nA}$ ). The movie sequence is available at http://www.tn.utwente.nl/tomvs/ projecten/project9harold.htm on the Internet.

and the CC cluster changes from an odd number (17) to an even number $(16)$ and due to the local $c(4 \times 2)$ pinning it becomes impossible for the complete enclosed area between to exhibit the $c(4 \times 2)$ buckling registry. Between the $\mathrm{C}$ dimer and the $\mathrm{CC}$ cluster there is an area where the dimers do not know whether to align with the $\mathrm{C}$ dimer or the $\mathrm{CC}$ cluster. In addition, the complete $c(4 \times 2)$ region in the direct proximity of the trough dimer has changed its phase (atoms that buckle initially downwards now buckle upwards and vice versa). In image 3(f) the trough dimer has moved one position away from the $\mathrm{CC}$ cluster and now the number of dimers trapped between the $\mathrm{CC}$ cluster and the $\mathrm{C}$ dimer is odd again, allowing a complete $c(4 \times 2)$ buckling registry. Counting of the number of dimers trapped between $\mathrm{C}$ dimers and $\mathrm{CC}$ clusters shows that there is a distinct preference for an odd number of dimers (111 odd vs 15 even) allowing the development of a perfect $c(4 \times 2)$ ordering. As the free energies per unit area of the $(2 \times 1)$ and $c(4 \times 2)$ are about the same at room temperature [12] the preference for a perfect $c(4 \times 2)$ buckling registry rather than a $c(4 \times 2) /(2 \times 1)$ buckling registry must be due to the boundary formation free energy between the $c(4 \times 2)$ and $(2 \times 1)$ domains [18].

In Fig. 4 a schematic diagram of the buckling registry in the proximity of an adsorbed $\mathrm{C}$ dimer is depicted. The dimers in the proximity of the adsorbed $\mathrm{C}$ dimer exhibit two common features: (1) they induce locally pinned $c(4 \times 2)$ buckling and (2) the atoms of the four surface dimers that lie around the adsorbed $\mathrm{C}$ dimer all buckle downwards, i.e., towards the substrate. If an adsorbed $\mathrm{C}$ dimer moves to a neighboring trough position the buckling orientation of most dimers (two dimers do not change their orientation) in the proximity changes (atoms that buckle upwards before the diffusion event buckle inwards after the diffusion event and vice versa). For a double jump of a $\mathrm{C}$ dimer along the trough only four substrate dimers change their buckling registry. In retrospect this concerted motion of substrate atoms [change of buckling registry or conversion from $c(4 \times 2)$ to $(2 \times 1)$ ] during diffusion is not unexpected at all but is a direct consequence the local pinning of the surface dimers in the $c(4 \times 2)$ registry. Although trough diffusion has been observed for $\mathrm{Si}$ trough dimers on $\mathrm{Si}(001)$ the resolution was insufficient to resolve a possible concerted motion of atoms (dimers) in the direct proximity of the diffusing dimer [6]. Interestingly, Borovsky, Krueger, and Ganz [19] showed that for diffusion of a $\mathrm{Si} \mathrm{C}$ dimer on $\mathrm{Si}(001)$ along the trough the two atoms separate completely and move independently before rejoining in a new location. We do not have any direct evidence whether or not such a dissociation step is also present during the diffusion of a trough $\mathrm{Ge} \mathrm{C}$ dimer along the trough.

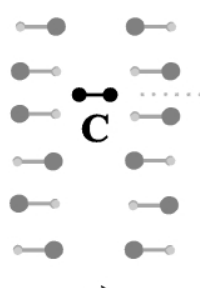

a)

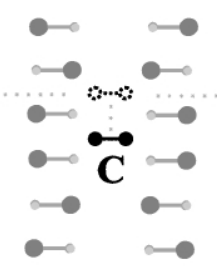

b)

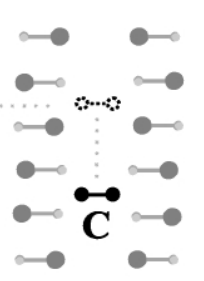

c)
FIG. 4. Schematic diagram of a diffusing $\mathrm{C}$ dimer along the trough $[(a),(b)$ single jump along the trough and (a)-(c) double jump along the trough]. Large and small circles represent atoms that buckle upwards and downwards, respectively. 
In conclusion, we have identified the various diffusion pathways for a $\mathrm{Ge}$ dimer on $\mathrm{Ge}(001)$ at room temperature. Moreover, we show that the diffusion of a trough $\mathrm{Ge}$ dimer along the trough goes along with a concerted motion of substrate atoms in the proximity of the diffusing dimer. This concerted motion of substrate atoms can easily be understood in terms of a pinning of the substrate in a $c(4 \times 2)$ registry in the proximity of the trough dimer.

H. J. W.Z. and E. Z. acknowledge support by the Netherlands Organization for Scientific Research (NWO).

* On leave from the University of South Australia, Mawson Lakes, Adelaide 5095 S.A. Australia.

[1] D. W. Bassett and P. R. Webber, Surf. Sci. 70, 520 (1978).

[2] G. L. Kellogg and P. J. Feibelman, Phys. Rev. Lett. 64, 3143 (1990).

[3] C. L. Chen and T. T. Tsong, Phys. Rev. Lett. 64, 3147 (1990).

[4] D. Dijkkamp, E. J. van Loenen, and H. B. Elswijk, in Ordering at Surfaces and Interfaces, edited by A. Yoshimori, T. Shinjo, and H. Watanabe (Springer-Verlag, Berlin, Heidelberg, 1992), p. 85.

[5] B. S. Swartzentruber, Phys. Rev. Lett. 76, 459 (1996).

[6] B. Borovsky, M. Krueger, and E. Ganz, Phys. Rev. Lett. 78, 4229 (1997).

[7] W. Wulfhekel, B. J. Hattink, H. J. W. Zandvliet, G. Rosenfeld, and B. Poelsema, Phys. Rev. Lett. 79, 2494 (1997).

[8] M. Krueger, B. Borovsky, and E. Ganz, Surf. Sci. 385, 146 (1997).
[9] E. Zoethout, H. J. W. Zandvliet, W. Wulfhekel, G. Rosenfeld, and B. Poelsema, Phys. Rev. B 58, 16167 (1998).

[10] Z. Zhang, F. Wu, H. J. W. Zandvliet, B. Poelsema, H. Metiu, and M. G. Lagally, Phys. Rev. Lett 74, 3644 (1995).

[11] B. S. Swartzentruber, A. P. Smith, and H. Jónsson, Phys. Rev. Lett. 77, 2518 (1996).

[12] H. J. W. Zandvliet, B. S. Swartzentruber, W. Wulfhekel, B. J. Hattink, and B. Poelsema, Phys. Rev. B 57, 6803 (1998); H. J. W. Zandvliet and B. Poelsema, Phys. Rev. B 59, 7289 (1999).

[13] H. Shigekawa, K. Hata, K. Miyake, M. Ishida, and S. Ozawa, Phys. Rev. B 55, 15448 (1997).

[14] X. R. Qin and M. G. Lagally, Phys. Rev. B 59, 7293 (1999).

[15] K. Hata, S. Yasuda, and H. Shigekawa, Phys. Rev. B 60, 8164 (1999).

[16] G. Brocks and P. J. Kelly, Phys. Rev. Lett. 76, 2362 (1996).

[17] X. R. Qin and M. G. Lagally, Science 278, 1444 (1997).

[18] The estimate of this free energy difference $(\sim 50 \mathrm{meV})$ does not include an effect of the size of the region undergoing the transition. In Ref. [12] it has been suggested that (1) the free energies per unit area for the $(2 \times 1)$ reconstruction and the $c(4 \times 2)$ reconstruction are comparable at room temperature and (2) the boundary free energy between a $(2 \times 1)$ and a $c(4 \times 2)$ domain parallel to the substrate rows is negligible. The estimated free energy difference therefore reflects the boundary free energy between a $(2 \times 1)$ and a $c(4 \times 2)$ domain in a direction perpendicular to the substrate dimer rows $(\sim 50 \mathrm{meV}$ per $32 \AA$, i.e., $1.6 \mathrm{meV} / \AA$ ).

[19] B. Borovsky, M. Krueger, and E. Ganz, Phys. Rev. B 59, 1598 (1999). 